\title{
T2API: Synthesizing API Code Usage Templates from English Texts with Statistical Translation
}

\author{
Thanh Nguyen ${ }^{1}$, Peter C. Rigby ${ }^{2}$, Anh Tuan Nguyen ${ }^{1}$, \\ Mark Karanfil ${ }^{2}$, Tien N. Nguyen ${ }^{3}$ \\ ${ }^{1}$ lowa State University, USA, ${ }^{2}$ Concordia University, Canada, ${ }^{3}$ University of Texas at Dallas \\ thanhng@iastate.edu,peter.rigby@concordia.ca,ntanhbk44@gmail.com, \\ mar_kar@encs.concordia.ca,tien.n.nguyen@utdallas.edu
}

\begin{abstract}
In this work, we develop T2API, a statistical machine translationbased tool that takes a given English description of a programming task as a query, and synthesizes the API usage template for the task by learning from training data. T2API works in two steps. First, it derives the API elements relevant to the task described in the input by statistically learning from a StackOverflow corpus of text descriptions and corresponding code. To infer those API elements, it also considers the context of the words in the textual input and the context of API elements that often go together in the corpus. The inferred API elements with their relevance scores are ensembled into an API usage by our novel API usage synthesis algorithm that learns the API usages from a large code corpus via a graph-based language model. Importantly, T2API is capable of generating new API usages from smaller, previously-seen usages.
\end{abstract}

\section{CCS Concepts}

-Software and its engineering $\rightarrow$ Software libraries and repositories; Integrated and visual development environments;

\section{Keywords}

Text-to-Code Translation, API Usage Synthesis, Graph-based Statistical Machine Translation

\section{INTRODUCTION}

In software development, integrate development environments (IDEs) play an important role in helping developers in improving their productivity. Several aspects of IDEs need to be improved to better support developers [7]. Crucial features in IDEs are code completion and API recommendation. Developers type in partial code and after requested, the IDE suggests a list of candidate code tokens or code fragments. In this work, we introduce T2API, an API suggestion plugin for Eclipse IDE that receives as an input a short textual description of a programming task and suggests a code template of API usage that is likely to be used in realizing the task.

Permission to make digital or hard copies of all or part of this work for personal or classroom use is granted without fee provided that copies are not made or distributed for profit or commercial advantage and that copies bear this notice and the full citation on the first page. Copyrights for components of this work owned by others than ACM to post on servers or to redistribute to lists, requires prior specific permission and/or a fee. Request permissions from Permissions@acm.org.

FSE'16, November 13-18, 2016, Seattle, WA, USA

(C) 2016 ACM. 978-1-4503-4218-6/16/11 ...\$15.00

http://dx.doi.org/10.1145/2950290.2983931
The code template is synthesized via a statistical learning process. The resulting API usage template, i.e., the combination of API elements (API method calls and classes) is synthesized from the API elements or smaller API usages that exist in the training code corpus. However, as a whole, the synthesized usage might not exist in the corpus. This service complements well to the traditional code completion when a programmer does not know API usages involving multiple APIs, thus leading to API failures [13].

T2API is designed following statistical machine translation (SMT) approach [14 15] in natural language processing (NLP). SMT statistically learns the translation "rules" from a parallel corpus of translation examples and applies the learned rules to translate a sentence $s$ from the source language $\left(L_{S}\right)$ to the target one $\left(L_{T}\right)$. A SMT model has three key components: the translator (also called a decoder) which uses a mapping model $(M)$ and a language model (LM) for the target language $\left(L_{T}\right)$ to translate $s$ into a sentence $t$ in $L_{T}$.

First, the goal of the mapping model $M$ is to learn a conditional probability for any pair of words/phrases in the two languages. Second, the language model learns the occurrence probability for any target sentence. Such model is useful in producing a natural sentence in the target language. A popular LM is the $n$-gram model [18]. For translation, the translator uses the mapping model and the language model $L M$ to compute the likelihood for each candidate sentence $t$ in $L_{T}$. That likelihood is based on the likelihoods of the aligned words/phrases in $s$ and $t$ according to $M$, and on the occurrence probability of the sentence $t$ in $L_{T}$ according to $L M$. Mathematically, the translator aims to find the best translation sentence in $L_{T}$ in the following sense: $\arg \max _{t \in L_{T}} P(t \mid s)=\arg \max _{t \in L_{T}} P(s \mid t) \times P(t)$. The mapping model is responsible for estimating $P(s \mid t)$ for the pairs of $s$ and $t$ in two languages while the language model computes $P(t)$ of the sentences $t$ in the target language.

For example, the following sentence in German "Morgen fliege ich nach Indien fur eine Konferenz" is translated into "Tomorrow I fly to a conference in India" in English. In the first step (called the Mapping step), the mapping model provides the mappings with the likelihoods for the pairs of words/phrases. For example, "Morgen" becomes "Tomorrow", "fliege" becomes "fly", "ich" becomes "I", etc. If more words are needed, SMT could have a second step, called Addition, to include additional words in the target language. In the final step, called Re-ordering, the words could be reordered if needed, for example, between "fly" and "I" in the final result.

\section{APPROACH OVERVIEW}

In this work, we aim to translate English input into an API code usage. For example, given the input "send network messages", we want to produce the API elements Socket.open, Message.compose, Message.send, and Socket.close. A possible solution is to directly ap- 
ply the aforementioned SMT model as in a related tool, SWIM [25]. However, SWIM would face the following challenges.

First, the left-to-right order in NLP translation might not be efficient due to the large numbers of mapped APIs that need to be considered at each step. Moreover, there exist important API elements which affect the selections of other APIs during translation. For example, if we map "send" first, there would be many choices for it such as Printer.send, MailServer.send, etc. However, if "messages" is selected for mapping first, many of such irrelevant choices for "send" can be eliminated. The choice Message.send would be ranked higher. In other words, the order of mapping is important for an efficient translation algorithm.

Second, in API usage generation, the re-ordering step requires a language model that supports the partial orders among API elements in API usages. The order of API elements depends on their data and control dependencies in API usages. Such orderings among some API code elements are strictly required while others can appear before or after each other. For example, a Socket object needs to be instantiated (via Socket.open) before it can be used and then closed via Socket.close. However, to open two sockets for reading and writing, one can instantiate either Socket object first.

To address the first challenge, in T2API, we develop an contextual expansion algorithm that uses the IBM Model [8] to map the words into API elements with a prioritized order. Generally, for an input text, to generate API elements, we take into account the dependencies of its words. To determine the next word for mapping, we consider the already-translated words because they help distinguish the context for that word. For example, "send" should be mapped to Message.send (rather than Printer.send) because "messages" has already been translated. To determine an API element among several alternatives, we consider also the already-generated API elements because they provide the context to decide better which element should be selected for the current word. Let us call the first context word context and the second one code context. Specifically, in training, we use IBM Model to learn the mappings between words and API elements from a StackOverflow corpus. For mapping, the words determined as having higher priority (e.g. "messages") will be mapped first using the trained IBM Model. Then, T2API gradually expands to map the lower priority words. The priorities of words are determined based on both contexts. For example, Socket.open is selected due to its relevancy with the word "network" and the already-mapped element Message.send.

To address the ordering challenge, we develop a usage graph synthesis algorithm. It uses a graph representation [23], called a usage graph, to represent data and control dependencies among API elements in usages. To estimate the occurrence likelihood of an API usage graph, it uses a graph-based language model called GraLan [22]. Our graph synthesis uses a beam search strategy to synthesize a new usage graph that covers the mapped API elements and maximizes the likelihoods of those elements being put in certain order computed by GraLan (e.g., Socket.new must come before Socket.close).

\section{T2API'S ARCHITECTURE}

This section explains the general architecture of T2API (Figure 1) with its two operational phases of training and translating.

\subsection{Training}

The left-hand side of Figure 1 shows the training process of our model. Two key building blocks are the mapping and language models, which need to be trained before translation.

Mapping Model. The goal of the mapping model is to learn the mappings between individual words and API elements. We make

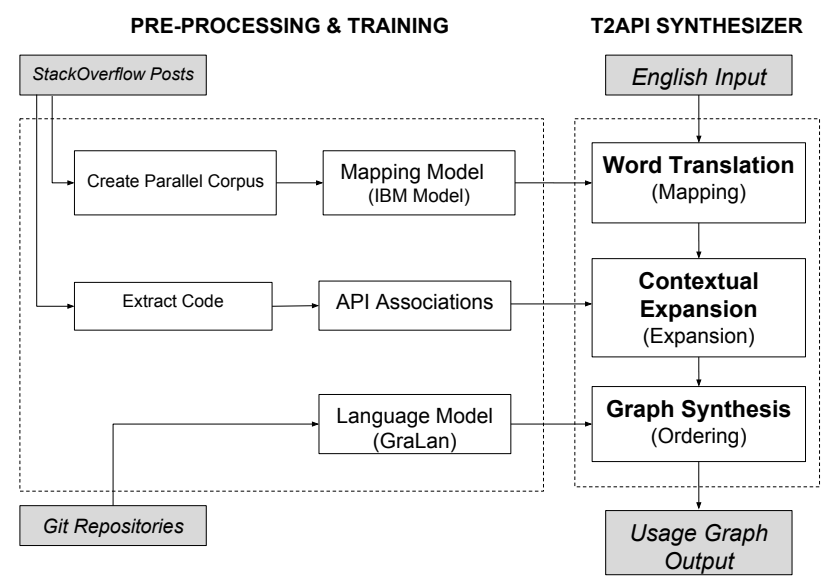

Figure 1: T2API Architecture

use of the IBM Model [8] for this word-to-API mapping task. The principle of IBM Model is to learn the mapping pairs via maximizing the likelihoods of observing them over a large number of pairs of English texts and corresponding sequences of APIs in a parallel corpus. For example, a description "write to a socket" corresponds to the API elements Socket.new, Socket.write, and Socket.close.

ACE tool to process SO posts. To build such training pairs for IBM Model, we processed a large number of StackOverflow (SO) posts using ACE tool [27], which is similar to island grammar technique used in [5]. We process the SO posts with answers having high ratings and corresponding code snippets. We remove stopwords (a, the, etc.) from the posts and extract keywords/keyphrases (copy, file, save, etc.). ACE extracts API elements embedded within freeform text. ACE can identify type and package information from API elements in freeform texts and from incomplete code snippets.

Then, we collect pairs of the textual descriptions on programming tasks (without API elements) and the corresponding sets of the API elements realizing the tasks mentioned in the posts. Those API elements include the ones in the code snippets (if any) and those embedded within the texts. Those pairs are produced by Create Parallel Corpus module in Figure 1 and used to train the IBM Model. After training, the model is able to provide the mappings between words and API elements. A word can be mapped to one or multiple APIs and vice versa. For a word, we have a ranked list of API elements with their relevance scores.

The ACE tool was also used to extract the API elements that co-occur in the posts. The associations among API elements are used by the expansion algorithm in considering the code context.

Language Model. The language model in SMT is used to estimate how likely a sentence occurs in the target language. In T2API, we use GraLan [22], a graph-based LM for API usages, that supports the modeling of API usages via graphs. The rationale of our choice is as follows. GraLan uses a usage graph representation [23], which was shown to better capture the data and control dependencies among API elements in API usages than the phrase-based models (e.g., ngram model) [22]. The reasons are, first, the API elements in a usage might not need to follow a strict order as enforced in phrase-based models. Second, the related elements of an API usage can be far apart. Sequences of tokens with limited sizes cannot recognize those usages. Third, a phrase-based model is based on previous elements, thus, it may not capture correct API usages due to irrelevant tokens.

For illustration, Figure 3 partially shows the API usage graph [23] for the code in Figure 2 An API usage graph [23] is a graph in which the nodes represent API object instantiations, variables, API 
Answer: (Rating 132)

To copy a file and save it to destination path you can use the method below.

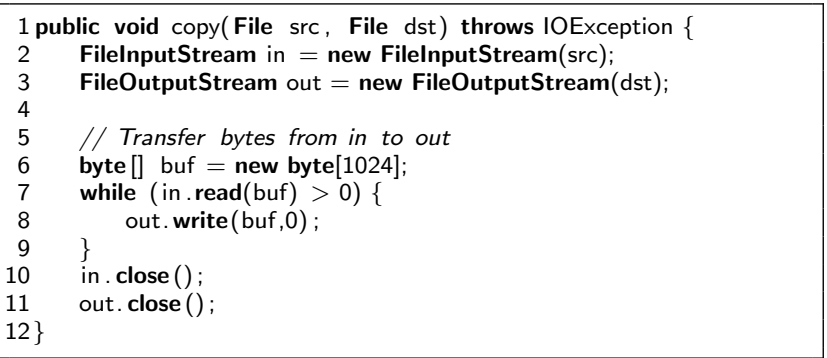

Figure 2: StackOverflow Answer 9292954

calls, field accesses, and control points (i.e., branching points of control units, e.g., if, while, for). The edges represent the control and data dependencies between the nodes. The node WHILE represents the loop control unit. Details on API usage graphs are in [23].

The graph language model [22] is built for API usage graphs. When being trained on a code corpus, GraLan first builds API usage graphs for all the methods in the projects in the corpus. For the entire dataset of the API usage graphs, it then calculates all possible subgraphs and estimates how likely it is for a certain node (an API element) to be connected to a given API usage graph via inducing edges. Details on training for GraLan can be found in another paper [22].

\subsection{Translation}

For translating a given English text, T2API operates in three steps: Mapping, Expansion, and Ordering (Figure 1).

Mapping. For mapping, it uses the trained IBM Model to derive the ranked list of API elements for each word in the given text.

Expansion. The goal of expansion is to collect the set of API elements most relevant for the given text. A naive solution is to collect the top-ranked API elements for each word into the resulting set. That solution faces two key challenges. First, a word can be used in different contexts with other words describing different programming tasks, and it can be mapped to multiple APIs. Thus, the already-translated words might provide contexts for expanding API elements from the current word. For example, the word "open" in "open a socket" should be mapped to Socket.open rather than FilelnputStream.open, if we know that "socket" is mapped to Socket.new. Second, an API can also be used in multiple contexts for different tasks, thus, it can be mapped to multiple words. The relevant API elements are inter-dependent of one another. A choice of mapping for an API could affect the mapping for the next API element. Due to those reasons, if we pick for a word the top-ranked (most frequent) respective API, it might not fit with the current text and code contexts.

We developed a contextual expansion algorithm to collect the set $S$ of relevant API elements. To account for contexts, we rely on two principles. First, to account for the dependency among selected API elements (code context), the next API element must have the highest relative co-occurrence frequency with all API elements that were already selected in the resulting set. The co-occurrence frequencies among API elements are computed by the API association module (Figure 1). The idea is that $c$ and $c^{\prime}$ (e.g., FilelnputStream.new and FilelnputStream.close) often going together indicates a certain relation in a usage. The chosen element must also have the highest mapping score with the currently translated word.

Second, to consider the word context in the given query, the next word $t$ to be translated must have the highest relative co-occurrence

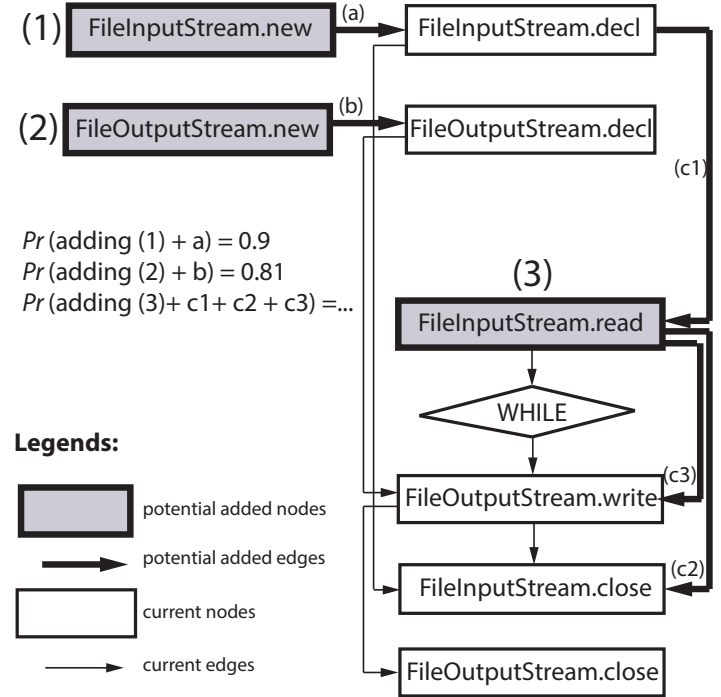

Figure 3: Graph Synthesis

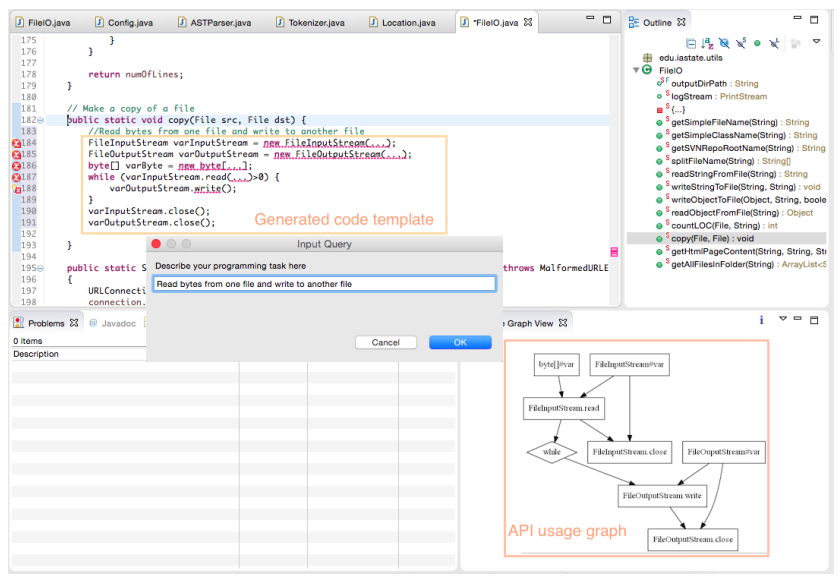

Figure 4: Synthesizing API Code Usage Template with T2API

frequency with all already-translated words. Our intuition is that the co-occurrence of $t$ and $t^{\prime}$ could be part of a description of a certain task (e.g., "open" and "file"). Finally, the elements in $S$ with their relevance scores are passed to the graph synthesizer (Ordering).

Ordering (Graph Synthesis). Our usage graph synthesizer synthesizes the API usage graph that covers as many relevant API elements produced after the expansion process as possible. For synthesis, we expand the graph one node at a time by finding the best API element to be added by maximizing

1) the likelihoods (computed by GraLan) of new API nodes being added to the graph, and

2) the relevance scores of the nodes to the query (from the mapping model).

Figure 3 illustrates the potential nodes (in gray color) and edges that could be added to the current API usage graph. For example, after training from the large code corpus, GraLan is able to estimate how likely the node FilelnputStream.new is added via the edge labeled (a), how likely the node FileOutputStream.new is added via the edge labeled (b), and how likely the node FilelnputStream.read is added via the edges $(\mathrm{c} 1),(\mathrm{c} 2)$, and $(\mathrm{c} 3)$.

We start with the nodes for the central API elements identified via the mapping model. Other nodes are gradually added based on their 


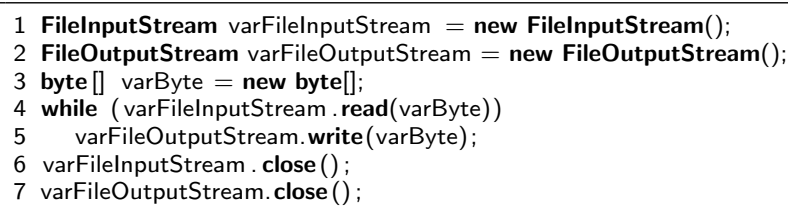

Figure 5: An Example of Generated JDK API Code Template

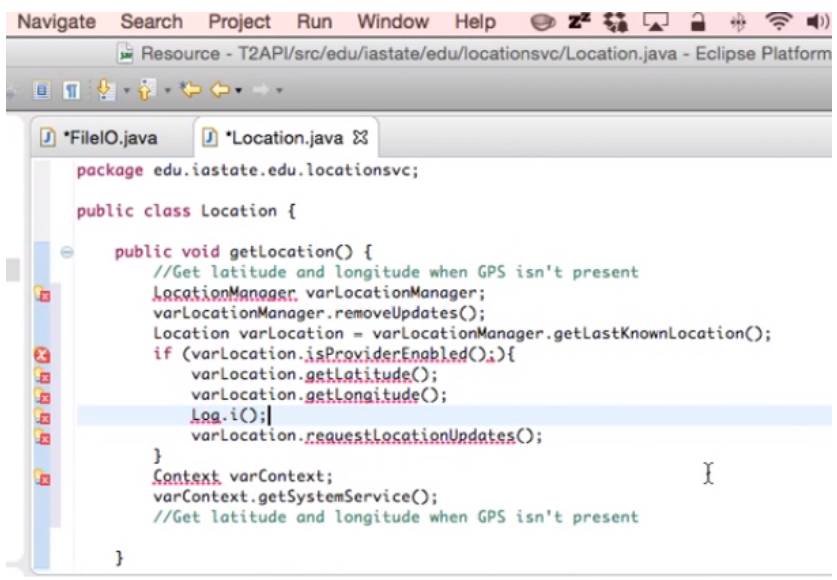

Figure 6: An Example of Generated Android API Template

likelihoods. We stop expanding if all the API elements produced in the first step are covered or the likelihood is lower than a threshold.

During synthesis, some sub-graphs of the newly expanded graph involving the new node and edges are seen in the corpus. However, the entire generated graph might be unseen in the corpus. Resulting synthesized graph or at least its smaller subgraphs (representing smaller usages) have high regularity, i.e., a high likelihood to be regularly used according to the corpus as well as high relevancy to the words in the query. Finally, the candidate API usage graphs are displayed in terms of textual templates as in Figure 5

\subsection{Tool Development}

We develop T2API, an Eclipse plugin that automatically translates from English input into API usages (Figure 4).

First, a user is working on their code within Eclipse environment. (S)he then wants to invoke T2API to suggest a template of an API usage for a specific programming task. For example, in Figure 4 a dialog window is open for a user to enter their description on "Read bytes from one file and write to another file". T2API will synthesize the API usage for the given query and use the top-ranked candidate in the current code. The query is used as the comment of the newly inserted API template. The user can modify the code to fit with his/her need.

T2API also displays in a separate window the API usage graph that shows the data and control dependencies among the API elements in the API template (Figure 4). Such information is valuable for the user to complete the template with concrete variables.

Figure 6 shows another usage scenario. A user enters the command "Get latitude and longitude when GPS isn't present". The tool produces the API code template as shown. This is an interesting case because the query comes from a real SO post \#11271458 [28]. In that post, the answer contains only the code snippet to use Geocoder to get the current location zip code. However, the author of that answer did not include the code to prepare a Location object to get the latitude and longitude to be used in the method
Geocoder.getFromLocation. In this case, T2API saw in the database that Geocoder.getFromLocation often goes with Location.getLongitude and Location.getLatitude, thus, synthesized them in a usage as shown in Figure 6 More information can be found in our website [29]. Our video demo can be found at https://www.youtube.com/watch ?v=Q8Kec6MHGY0\&feature=youtu.be

\section{RELATED WORK}

T2API is closely related to SWIM [25], which applies directly SMT to translate texts into API usages. SWIM synthesizes code by first using IBM Model to produce code elements and then using $n$-gram to synthesize a code sequence. In comparison, after IBM Model, we expand the set by using set translation. Also, we use GraLan as language model to synthesize graphs, which are suitable for API usages.

Maddison and Tarlow [16] present a generative model for source code, which is based on syntactic structures. TBCNN [21] also uses tree information for suggest next code tokens. In comparison, T2API uses GraLan, which can capture better program dependencies with graph structures. Allamanis et al. [4] introduce a jointly probabilistic model of short natural language utterances and source code snippets. In comparison, first, a joint model is used for both sides and a treebased representation is used for code and texts. T2API uses graph structures. Second, while their approach uses advanced bimodal modeling (e.g., image+text, text+code), we treat code synthesis as a machine translation problem, allowing different language models to be used for texts and source code. Anycode [10] uses a probabilistic context free grammar with tree representations for Java constructs and API calls to synthesize small Java expressions.

Statistical learning has been used in SE applications, including code suggestion [11], code and naming convention [1, 2], API suggestion [26], large-scale mining [3], effort estimation [20], etc. Information retrieval (IR) approaches. Code search engines match a query with keywords in program elements (Sourcerer [6]). Other group of IR-based code search approaches considers the relations among API elements in suggestion. McMillan et al. [19]'s approach first locates a set of APIs that are textually similar to the query and then finds code examples cover most of them. Chan et al. [9] consider the context of call graphs and API graphs when taking given texts as queries.

Constraint-based, program analysis approaches. Parseweb [30] uses flow analysis to suggest a sequence of API calls for a query with the input and output types. Prospector [17] is a technique for synthesizing jungloid code fragments given input/output types. In our work, FUSION [12]'s relationship-based constraints or object usage specifications in Pradel and Gross [24] could be also explored to support framework object usage recommendations.

\section{CONCLUSION}

We present a novel context-sensitive, graph-based statistical translation tool that takes a query and synthesizes complex API code templates and graphs with control units and dependencies among API elements. While we use texts from real-world StackOverflow with large numbers of words, the synthesized code captures more complex usages than the state-of-the-art sequence-based approaches. Our novel graph-based API synthesis algorithm generates the new graph representing an API usage from usages in a large code corpus.

\section{ACKNOWLEDGEMENTS}

This work was supported in part by the US National Science Foundation (NSF) grants CCF-1518897, CNS-1513263, CCF-1413927, CCF-1320578, and TWC-1223828. 


\section{REFERENCES}

[1] M. Allamanis, E. T. Barr, C. Bird, and C. Sutton. Learning natural coding conventions. In Proceedings of the International Symposium on Foundations of Software Engineering, FSE 2014, pages 281-293. ACM, 2014.

[2] M. Allamanis, E. T. Barr, C. Bird, and C. Sutton. Suggesting accurate method and class names. In Proceedings of the 10th Joint Meeting on Foundations of Software Engineering, ESEC/FSE 2015, pages 38-49. ACM, 2015.

[3] M. Allamanis and C. Sutton. Mining source code repositories at massive scale using language modeling. In Proceedings of the 10th Working Conference on Mining Software Repositories, MSR '13, pages 207-216. IEEE Press, 2013.

[4] M. Allamanis, D. Tarlow, A. Gordon, and Y. Wei. Bimodal modelling of source code and natural language. In Proceedings of the 32nd International Conference on Machine Learning, ICML'15. ACM, 2015.

[5] A. Bacchelli, A. Cleve, M. Lanza, and A. Mocci. Extracting structured data from natural language documents with island parsing. In Proceedings of the 2011 26th IEEE/ACM International Conference on Automated Software Engineering, ASE '11, pages 476-479. IEEE Computer Society, 2011.

[6] S. Bajracharya, T. Ngo, E. Linstead, Y. Dou, P. Rigor, P. Baldi, and C. Lopes. Sourcerer: A search engine for open source code supporting structure-based search. In Companion to the 21st ACM SIGPLAN Symposium on Object-oriented Programming Systems, Languages, and Applications, OOPSLA '06, pages 681-682. ACM, 2006.

[7] M. Beller, G. Gousios, A. Panichella, and A. Zaidman. When, how, and why developers (do not) test in their IDEs. In Proceedings of 10th Symposium on Foundations of Software Engineering, ESEC/FSE'15, pages 179-190. ACM, 2015.

[8] P. F. Brown, V. J. D. Pietra, S. A. D. Pietra, and R. L. Mercer. The mathematics of statistical machine translation: parameter estimation. Comput. Linguist., 19(2):263-311, June 1993.

[9] W.-K. Chan, H. Cheng, and D. Lo. Searching Connected API Subgraph via Text Phrases. In Proceedings of the 20th International Symposium on the Foundations of Software Engineering, FSE '12, pages 10:1-10:11. ACM, 2012.

[10] T. Gvero and V. Kuncak. Synthesizing java expressions from free-form queries. In Proceedings of the 2015 ACM SIGPLAN International Conference on Object-Oriented Programming, Systems, Languages, and Applications, OOPSLA 2015, pages 416-432. ACM, 2015.

[11] A. Hindle, E. T. Barr, Z. Su, M. Gabel, and P. Devanbu. On the naturalness of software. In Proceedings of the 2012 International Conference on Software Engineering, ICSE 2012, pages 837-847. IEEE Press, 2012.

[12] C. Jaspan and J. Aldrich. Checking framework interactions with relationships. In European Conference on ObjectOriented Programming, pages 27-51. Springer-Verlag, 2009.

[13] M. Kechagia, D. Mitropoulos, and D. Spinellis. Charting the API minefield using software telemetry data. Empirical Softw. Engg., 20(6):1785-1830, Dec. 2015.

[14] P. Koehn. Statistical Machine Translation. Cambridge University Press, New York, NY, USA, 1st edition, 2010.

[15] P. Koehn, F. J. Och, and D. Marcu. Statistical phrase-based translation. In Proc. of the Conference of the North American Chapter on Human Language Technology, NAACL'03, pages
48-54. Association for Computational Linguistics, 2003.

[16] C. J. Maddison and D. Tarlow. Structured generative models of natural source code. In Proceedings of the 31st International Conference on Machine Learning (ICML), June 2014.

[17] D. Mandelin, L. Xu, R. Bodík, and D. Kimelman. Jungloid mining: Helping to navigate the API jungle. In $A C M$ SIGPLAN Conference on Programming Language Design and Implementation, PLDI '05, pages 48-61. ACM, 2005.

[18] C. D. Manning and H. Schütze. Foundations of Statistical Natural Language Processing. MIT Press, Cambridge, MA, USA, 1999.

[19] C. McMillan, D. Poshyvanyk, and M. Grechanik. Recommending source code examples via API call usages and documentation. In Proceedings of the 2nd International Workshop on Recommendation Systems for Software Engineering, RSSE'10, pages 21-25. ACM, 2010.

[20] L. L. Minku and X. Yao. How to make best use of cross-company data in software effort estimation? In Proceedings of the 36th International Conference on Software Engineering, ICSE'14, pages 446-456. ACM, 2014.

[21] L. Mou, G. Li, Z. Jin, L. Zhang, and T. Wang. TBCNN: A tree-based convolutional neural network for programming language processing. CoRR, abs/1409.5718, 2014.

[22] A. T. Nguyen and T. N. Nguyen. Graph-based statistical language model for code. In Proceedings of the 37th International Conference on Software Engineering, ICSE 2015. IEEE CS, 2015.

[23] T. T. Nguyen, H. A. Nguyen, N. H. Pham, J. M. Al-Kofahi, and T. N. Nguyen. Graph-based mining of multiple object usage patterns. In Proceedings of Foundations of Software Engineering, ESEC/FSE '09, pages 383-392. ACM, 2009.

[24] M. Pradel and T. R. Gross. Automatic generation of object usage specifications from large method traces. In Proceedings of the IEEE/ACM International Conference on Automated Software Engineering, ASE '09, pages 371-382. IEEE, 2009.

[25] M. Raghothaman, Y. Wei, and Y. Hamadi. Swim: Synthesizing what i mean: Code search and idiomatic snippet synthesis. In Proceedings of the 38th International Conference on Software Engineering, ICSE '16, pages 357-367. ACM, 2016.

[26] V. Raychev, M. Vechev, and E. Yahav. Code completion with statistical language models. In Proceedings of the 35th ACM SIGPLAN Conference on Programming Language Design and Implementation, PLDI '14, pages 419-428. ACM, 2014.

[27] P. C. Rigby and M. P. Robillard. Discovering essential code elements in informal documentation. In Proceedings of the 2013 International Conference on Software Engineering, ICSE '13, pages 832-841. IEEE Press, 2013.

[28] StackOverflow. http://stackoverflow.com/questions/11270229/how-to-usegeocoder-to-get-the-current-location-zipcode/11271458\#11271458.

[29] T2API Website. http://home.engineering.iastate.edu/ anhnt/Research/T2API/

[30] S. Thummalapenta and T. Xie. Parseweb: A programmer assistant for reusing open source code on the web. In Proceedings of International Conference on Automated Software Engineering, ASE '07, pages 204-213. ACM, 2007. 